\title{
Shh Overexpression Is Correlated with GRP78 and AR Expression in Primary Prostate Cancer: Clinicopathological Features and Outcomes in a Chinese Cohort
}

This article was published in the following Dove Press journal:

Cancer Management and Research

\author{
Xiangyu Zhang $\mathbb{D}^{1, *}$ \\ Yanmin Zhang ${ }^{2, *}$ \\ Fanzhong Lin' \\ Xin Shi ${ }^{1}$ \\ Longquan Xiang ${ }^{\prime}$ \\ Liang $\mathrm{Li}^{\prime}$ \\ 'Department of Pathology, Jining First \\ People's Hospital, Jining Medical \\ University, Jining 272000, People's \\ Republic of China; ${ }^{2}$ Department of \\ Pathology, Gaomi People's Hospital, \\ Gaomi 26I500, People's Republic of \\ China
}

*These authors contributed equally to this work
Correspondence: Longquan Xiang; Liang Li Department of Pathology, Jining First People's Hospital, Jining Medical University, No. 6 Jiankang Road, Jining 272000, People's Republic of China

Tel/Fax +86537 605I547

Email xianglongquan0I@163.com;

liliang0II@I26.com
Introduction: Shh plays an important role in prostate cancer progression, but its correlation with GRP78 and AR is elusive.

Methods: The study included 539 patients in total, of which 443 had primary prostate carcinoma and 96 patients had benign prostatic hyperplasia (BPH). The clinicopathologic features, histologic scores of protein expression, and correlations between protein and disease state were studied in this cohort. Kaplan-Meier and Pearson correlation analyses were used to compare measures between groups. We performed immunohistochemistry to evaluate the expression of the Shh protein in benign prostatic hyperplasia $(n=96)$ and prostate cancer (Gleason scores $\leq 6[n=399]$ or $\geq 7[n=44]$ ). We quantified the expression of Shh, AR, and GRP78 using the weighted histoscore method, studied the correlation between Shh expression and AR and GRP78, and evaluated the impact of Shh protein expression on patient survival.

Results: Shh expression was significantly higher in prostate cancer with Gleason scores $\geq 7$ than in cancer with lower Gleason scores or benign hyperplasia and was much higher in ARpositive cancer than in AR-negative cancer. Shh is overexpressed in high-grade prostate cancer and is positively correlated with the expression of both GRP78 and AR.

Conclusion: Therefore, Shh may be a useful prognostic marker and therapeutic target for prostate cancer.

Keywords: prostate cancer, GRP78, Shh, AR, survival analysis

\section{Introduction}

Prostate cancer is one of the most commonly diagnosed cancers both in China and worldwide and is the second leading cause of cancer-related deaths in the USA. ${ }^{1,2}$ The increasing prevalence of prostate cancer has made it a growing public health concern in China. Early diagnosis and effective treatment of prostate cancer have largely increased the five-year survival rates for prostate cancer. ${ }^{3}$ The widespread use of prostate-specific antigen (PSA) testing enables some men to be diagnosed with very early-stage prostate cancer; therefore, clinical use of this testing in screening, diagnosis, prognostic stratification increases the survival rate of prostate cancer patients. ${ }^{4,5}$ However, benign prostate hyperplasia and other urologic diseases also lead to serum PSA changes. Histopathological diagnosis is the "golden standard" for prostate cancer diagnosis. Gleason score is the most widely used grading system for prostate cancer, it can 
effectively predict the clinical outcome of patients. Gleason scoring on a prostate-cancer histopathological examination is also very important for predicting patient survival and is one of the prognostic indicator for prostate cancer patients. ${ }^{6,7}$ However, the Gleason grading system is based on subjective judgments, which may lead to varied results among different observers. Further, due to the limitations of biopsy sampling, biopsy grading may be inconsistent with the prostatectomy specimen. ${ }^{8,9}$ Therefore, prostate cancer patients with apparently identical morphologies may have different survival rates, partly due to variable subjective observations. However, generally, patients with higher Gleason scores usually have worse prognoses than those with low scores. ${ }^{10}$

In recent years, immunohistochemistry (IHC) plays an important role in cancer clinical diagnosis. IHC has brought revolutionary changes to traditional clinical pathology and has become a powerful tool for clinical pathological diagnostics. ${ }^{11,12}$ Several important biomarkers such as AR, Shh, GRP78, PSA, P63, P504S were usually detected using the IHC method for clinical pathologic diagnosis. The prostate is composed of epithelial glands and fibromuscular stroma, and its main function is to secrete seminal fluid. And prostate adenocarcinoma usually arises from tissues of the epithelial gland. Epithelial cells in the prostate secrete prostatic fluid and PSA in an androgen-dependent manner. Epithelial cells usually express the androgen receptor (AR), and their growth can be stimulated by binding with androgen. Similar to normal prostate epithelial cells, prostate cancer cells also require androgen to grow and survive. Therefore, orchiectomies, which lead to androgen deprivation, can retard prostate cancer growth. However, androgen receptor variants V7 and V9 have been shown to be associated with drug resistance. ${ }^{13,14}$ In a normal prostate, the stroma is also androgen-responsive. Fibroblasts, smooth muscle cells, endothelial cells, and infiltrating cells can produce growth factors that provide paracrine signals to the prostate epithelial cells. However, in prostate cancer, the stroma is altered and the extracellular matrix is remodeled, increasing angiogenesis and alters inflammatory cells. ${ }^{15}$ Thus, the stroma is reactivated, and bidirectional signaling between cancer and stromal cells can further accelerate the progression of prostate cancer. ${ }^{16,17}$ The Sonic hedgehog (Shh) protein probably plays some essential roles in this bidirectional signaling.

Shh protein is an important component of the Shh signaling pathway and participates in maintaining stem cell populations and repairing tissue damage. The Shh protein is also expressed by cancer cells and is secreted into the tumor microenvironment, thus stimulating the stromal cells to express more growth factors by activating the Shh signaling cascade and, in turn, stimulating cancer cell growth. ${ }^{18,19}$ Prostate cancer cells express more levels of Shh compared with normal prostatic epithelia, and Shh potentiates cancer cell growth. ${ }^{20}$ Shh signaling pathway components express mare typically expressed at much higher levels in advanced prostate cancer (Gleason scores 8-10) compared with lowgrade prostate cancer (Gleason scores 3-6). ${ }^{21}$ In addition, blockade of the Shh signaling pathway has been shown to lead to cancer shrinkage and remission in a xenograft model of prostate cancer. ${ }^{22}$ Recently, one study showed that a deficiency in Shh could lead to ER stress of Paneth cells in the intestines. ${ }^{23}$ Further, another recent study has suggested that ER stress could affect medulloblastoma tumorigenesis via Ptch1, a component of the Shh signaling pathway. ${ }^{24}$ Prostate cancer cells usually require increased endoplasmic reticular capacity and function to synthesize more proteins in the endoplasmic reticulum (ER). ER chaperones play important roles in maintaining ER homeostasis. The $78 \mathrm{kDa}$ glucose-regulated protein (GRP78)/heat-shock protein 5(HSPA5) is one of the most important ER chaperones and is involved in tumorigenesis and drug resistance in various cancers. ${ }^{25,26}$ We hypothesized that AR regulates the expression of Shh and GRP78 and that GRP78 could interact with Shh to regulate its expression.

In this study, we focused on three important proteins AR, Shh, and GRP78 - to evaluate their prognostic value in prostate cancer with different Gleason scores. Further, we investigated possible relationships between AR, Shh, and GRP78. This information could be of value in diagnosing prostate cancer patients and evaluating prognoses.

\section{Materials and Methods Ethical Standards}

The ethics committee of Jining First Peoples' Hospital, China, approved this study, and informed consent was written by all patients before being included in the study. The study was performed in accordance with the ethical standards of the 1964 Declaration of Helsinki and its later amendments.

\section{Patients}

The study included 443 patients with primary prostate carcinoma and 96 patients with benign prostatic hyperplasia (BPH). Prostate cancer (PCa) tissues were obtained during radical prostatectomies performed between 2000 and 2015 
at the Jining No. 1 Peoples' Hospital, China. All tissues were taken from prostate cancer patients at the time of transurethral prostate resection and were immediately fixed in $20 \%$ formalin. Two independent consultant pathologists determined the Gleason scores for all samples. Table 1 summarizes the clinicopathological data for the patient cohorts.

\section{Immunohistochemistry}

Paraffin-embedded tissue blocks were sectioned into $4-\mu \mathrm{m}$ slices, deparaffinized, and mounted on glass slides. Antigens were demasked in EDTA buffer ( $\mathrm{pH}$ 8.4). The primary antibodies used were specific for Shh (Cell Signaling; No. 2207; 1:50 dilution; we used a microwave and citrate buffer-based method for antigen retrieval), GRP78 (Santa Cruz Biotechnology H-129; sc-13539; 1:100 dilution citrate buffer and water-bath antigen retrieval), or AR (polyclonal rabbit Ig; Abcam, Cambridge, MA, USA). After incubation with the secondary antibody, the presence of antigen was visualized using 3,3-diaminobenzidine tetrahydrochloride (DAB), and the slides were counterstained with hematoxylin. To exclude nonspecific staining from the secondary antibodies, we tested negative controls that lacked the primary antibody.

Table I Clinicopathologic Characteristics of Patients Included in the Study

\begin{tabular}{|l|l|}
\hline Variable & No. Patients \\
\hline Malignant tissue & 443 \\
Benign tissue & 96 \\
Median age (range), year & $68(62-73)$ \\
Follow-up time (range), months & $117(43-265)$ \\
Median PSA at diagnosis, median (range), ng/mL & $8.4(4.1-47)$ \\
\hline Gleason score & No. \\
$2-6$ & $399(90.2 \%)$ \\
$\geq 3+4$ & $44(9.8 \%)$ \\
\hline Clinical stage & \\
TI & $341(77.0 \%)$ \\
T2 & $89(20.1 \%)$ \\
T3 & $13(2.9 \%)$ \\
\hline CRPC & \\
Yes & $146(41 \%)$ \\
No & $292(34 \%)$ \\
Not available & $5(1.1 \%)$ \\
\hline Overall death & \\
Yes & $138(31.1 \%)$ \\
No & $291(65.6 \%)$ \\
Not available & $14(3.1 \%)$ \\
\hline
\end{tabular}

\section{Expression Analysis}

Histoscores were applied to interpret the results. Briefly, staining intensity was graded as negative (0), weak (1), moderate (2) or strong (3), then the score was multiplied by the percentage of tumor cells within each category. ${ }^{27}$ The final histoscores ranged from 0 to 300 . Two observers, who were blinded to all outcome data, graded the GRP78/ $\mathrm{BiP}, \mathrm{AR}$, and Shh staining.

\section{Statistics}

Statistical analyses were performed using GraphPad Prism V (GraphPad Software, San Diego, CA, USA). Statistical data were evaluated using the Mann-Whitney test to compare differences in GRP78 expression among the different cancer samples. Disease-specific survival rates were generated using the Kaplan-Meier method. The survival curves were compared using the Log-rank test and correlations were calculated using the Pearson correlation coefficient. A result with $\mathrm{P}<0.05$ was considered statistically significant.

\section{Results}

\section{PCa Patient Cohort Characteristics}

Table 1 shows the patient and prostate cancer characteristics of the 443 PCa patients. The median age at the time of surgery was 61 years (range, 45-71 years). More than twothirds of patients had a clinical T1 stage, and only a few patients had a pathological Gleason score (GS) $\geq 8$. Median follow-up time was 117 months (range, 43-265 months), and $23 \%$ of patients developed biochemical recurrence (BCR) of PCa following radical surgery, which was defined as a confirmed postoperative PSA level $>4 \mathrm{ng} / \mathrm{mL}$. Shh expression was evaluated in both the benign and cancer tissue samples, in AR (+) and AR (-) cases, and in GRP78 $(+)$ and GRP78 (-) cases. The individual patients' Gleason scores and relevant protein expression levels of Shh, AR, and GRP78 were listed in Table S1.

\section{Protein Expression}

Shh, GRP78, and AR expressions were evaluated in the 443 cases of primary $\mathrm{PCa}$ and the 96 control benign prostatic hyperplasia (BPH) samples. Shh was mainly expressed in the extracellular matrix, with low signal in the nucleus or cell membrane. GRP78 was also mainly expressed in the cytoplasm but not in the cell membrane. AR was mainly expressed in the cell membrane, with low signal in the nucleus and cytoplasm (Figure 1). Shh signals in the PCa 
tissue (GS $\geq 7$ ) were much stronger than in the PCa tissue $(\mathrm{GS} \leq 6)$ and BPH samples $(\mathrm{P}<0.0001)$. The median histoscore was 58 for benign tissue, 172.8 for the malignant $\mathrm{PCa}$ tissue with $\mathrm{GS} \geq 7$, and 147.5 for the malignant PCa tissue with GS $\leq 6$ (Figure 2A). PCa patients were divided into $\mathrm{AR}^{+}$ and $\mathrm{AR}^{-}$groups based on $\mathrm{AR}$ expression, and $\mathrm{Shh}$

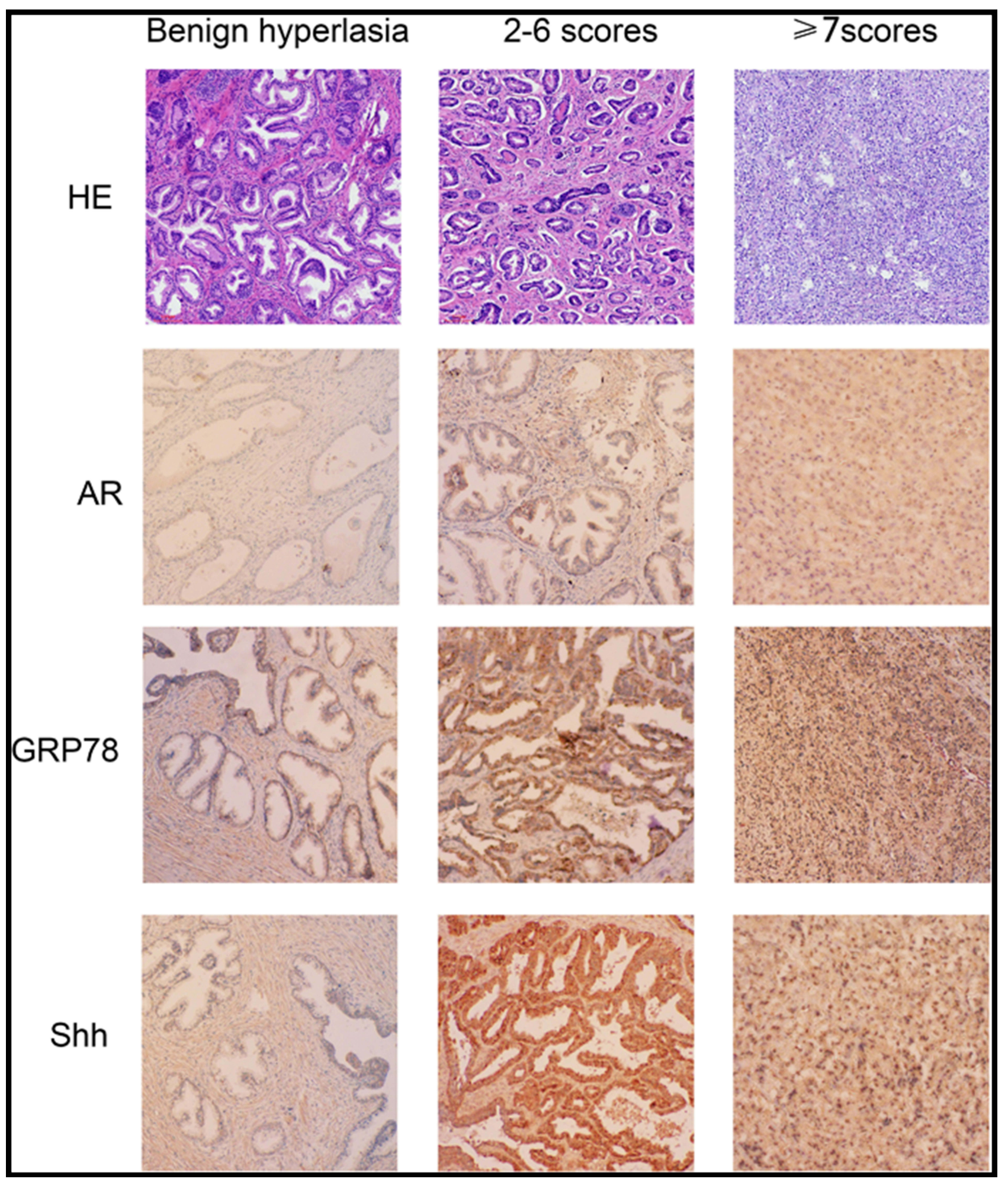

Figure I Shh, GRP78, and AR protein expression levels in benign prostate hyperplasia and prostate cancer (Gleason scores of 2-6 or 7-10) were evaluated via immunohistochemistry. 
expression was found to be much higher in the $\mathrm{AR}^{+}$group than in the $\mathrm{AR}^{-}$group, with histoscores of 154.8 and 88.0, respectively (Figure 2B). Similarly, the PCa patients were divided into GRP78 ${ }^{+}$and GRP78 ${ }^{-}$groups, and Shh expression in the GRP78 ${ }^{+}$group was found to be much higher than in the GRP78 ${ }^{-}$group, with histoscores of 168.5 and 102.7, respectively (Figure 2C). Therefore, elevated Shh expression was associated with increased expression of both AR and GRP78. Cytoplasmic GRP78 expression and membrane AR expression, in particular, were associated with Shh expression, with correlation coefficients of 0.227 and 0.334 , respectively (Table 2 ).

To evaluate the prognostic value of Shh, we conducted a survival analysis in this patient cohort. Shh overexpression in all PCa patients was associated with shorter diseasespecific survival in patients with $\mathrm{AR}^{+}$tumors (Figure 2D). In the $\mathrm{AR}^{+}$subgroup, the median survival time for patients with tumors that had overexpression of Shh was 6.9 years compared with 8.1 years for patients with low GRP78expressing tumors, which was statistically significant
A

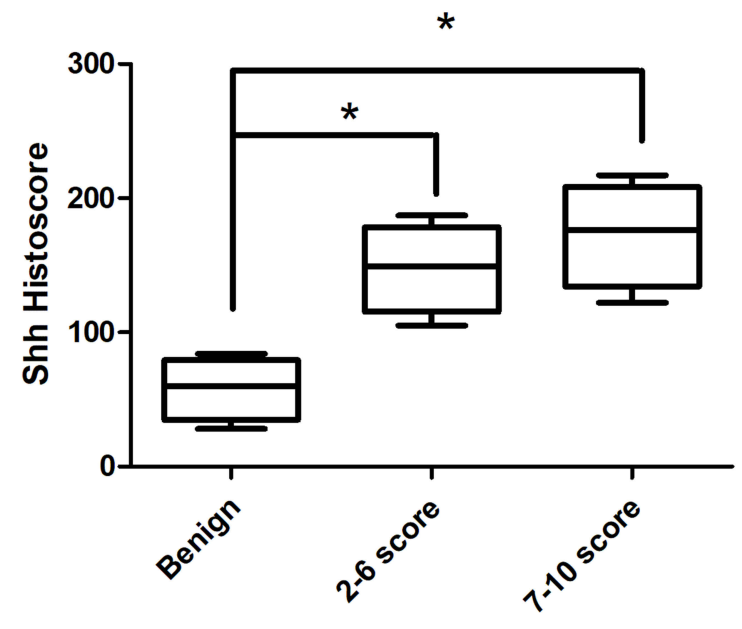

C

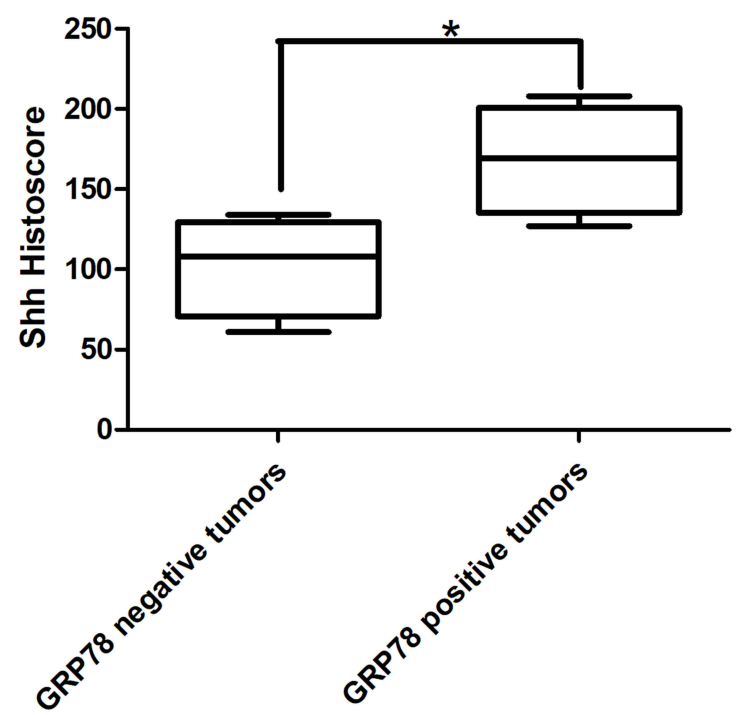

B

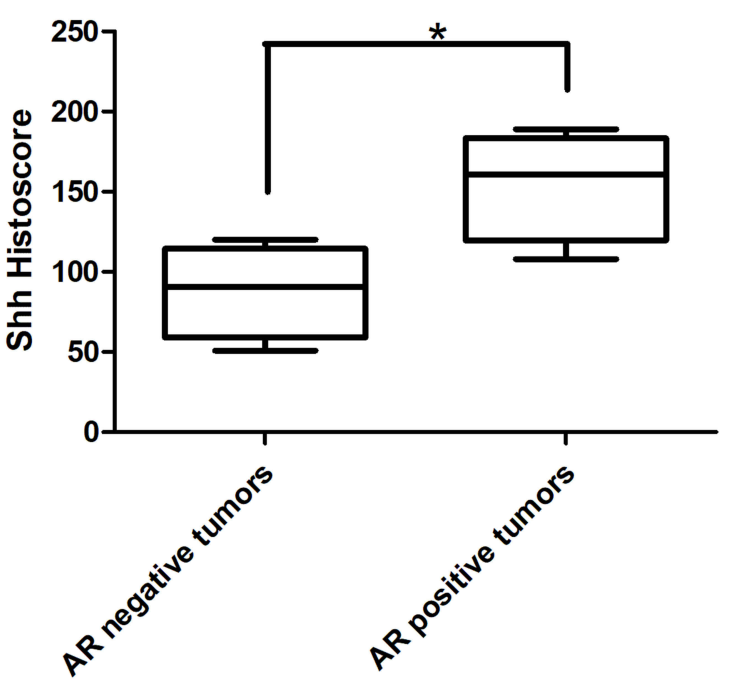

D

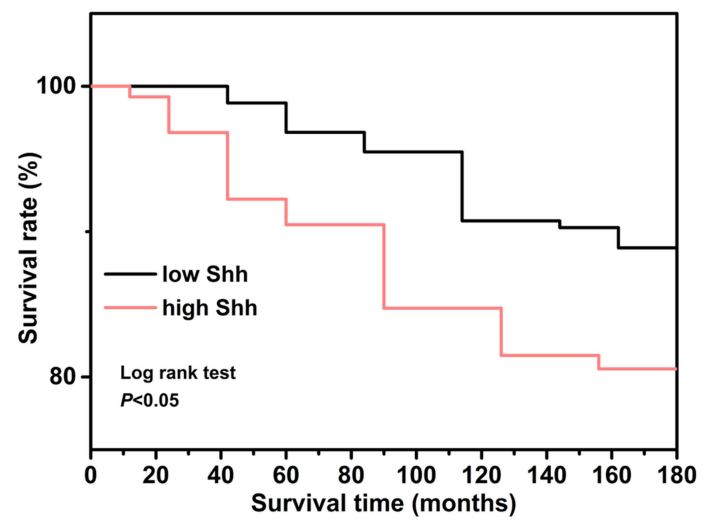

Figure 2 Shh expression levels in benign prostate hyperplasia and prostate cancer (Gleason scores of 2-6 or 7-10) (A). Difference in Shh expression between the ARpositive and AR-negative groups (B) and in the GRP78-positive and GRP78-negative groups (C). Disease-free survival analysis for AR-positive patients with Shh overexpression or with no expression (D). $* P<0.05$. 
Table 2 Association of Shh Expression with GRP78 and AR

\begin{tabular}{|l|l|l|}
\hline \multirow{2}{*}{} & \multicolumn{2}{|l|}{ Correlation to Shh Expression } \\
\cline { 2 - 3 } & Correlation Coefficients & Significance $\boldsymbol{p}$ values \\
\hline GRP78 & 0.227 & 0.001 \\
AR & 0.334 & 0.003 \\
\hline
\end{tabular}

$(\mathrm{P}<0.05)$. This correlation was not seen in $\mathrm{AR}^{-} \mathrm{PCa}$ patients $(\mathrm{P}>0.05)$ (Figure 3).

\section{Prostate Adenocarcinoma Data Analysis Using TCGA Database}

There was no significant difference in the expression of AR in normal or tumor samples ( $>0.05$ ); however, higher AR expression was associated with a shorter survival time, compared to low expression of AR ( $p>0.05)$. Across cancer subgroups (Gleason scores $=6,7,8,9,10$ ), in the high AR expression group, the group with Gleason score $=10$ had the shortest survival. The low/medium AR expression group had similar results, those with Gleason score $=10$ had the shortest survival, when compared with other groups $(\mathrm{p}<0.05)$ (Figure 4$)$.

The relative expression of GRP78/HSPA5 in normal versus tumor samples was not significantly different ( $\mathrm{p}>0.05$ ); however, higher GRP78/HSPA5 expression was associated with a shorter survival time compared with patients with low GRP78/HSPA5 expression ( $p>0.05)$. Across different cancer subgroups (Gleason scores $=6$, 7, $8,9,10)$, in patients with high GRP78/HSPA5 expression, those with Gleason score $=6$ had the shortest survival, in the GRP78/HSPA5 low/medium expression group, the

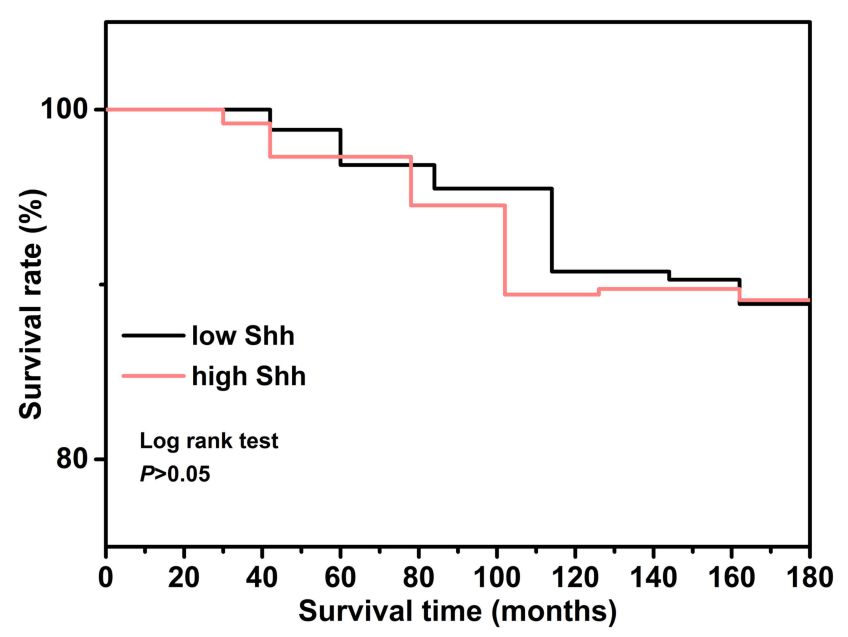

Figure 3 Disease-free survival analysis for AR-negative patients with Shh overexpression or no expression. group of Gleason score $=10$ had the shortest survival, when compared with other groups $(\mathrm{p}<0.05)$ (Figure 5).

The relative expression of Shh in normal versus tumor samples was not significantly different ( $p>0.05$ ), however higher Shh expression was associated with a shorter survival compared with the low Shh expression $(p>0.05)$; while across different cancer subgroups (Gleason scores $=6,7,8$, $9,10)$, in the Shh high expression group, the group of Gleason score $=10$ had the shortest survival, in the Shh low/medium expression group, the group of Gleason score $=9$ had the shortest survival when compared with other groups $(\mathrm{p}<0.05)$ (Figure 6).

\section{Discussion}

Initially, one study found that Hedgehog (Hh) was important in embryonic cell development. ${ }^{28}$ Recently, several studies have shown that an aberrant Hh signaling pathway contributes to cancer cell growth directly or indirectly via the tumor microenvironment. ${ }^{18,29}$ The regulation processes of the Shh-Gli signaling pathway are listed here: Shh is an Hh signaling pathway ligand, and when it binds to the Patched (Ptch) receptor expressed on the cell membrane, it attenuates the inhibitory effect on Smoothened (SMO), thus activating the Gli proteins. Gli proteins are important transcriptional factors that translocate to the nucleus after activation in the cytoplasm, and they regulate the expression of target genes, including Cyclin D1, Bcl-2, IL6, and PTHrP. ${ }^{30}$ Actually, Shh-Gli signaling pathway plays important roles in various types of cancer. Therefore, some targeted therapies have been developed to inhibit the Gli's transcriptional activity to treat cancer. ${ }^{31}$ Moreover, the Shh-Gli signaling pathway also exerts effects in cancer metastases and drug resistance, and downregulation of Shh could inhibit bone metastasis and partially reverse paclitaxel resistance in prostate cancer. And one study showed that inhibiting the Shh signaling pathway promotes docetaxel anti-cancer effects in prostate cancer. ${ }^{32}$ Cancer cells face a tough growth environment and are usually under chronic stress. Therefore, ER stress usually occurred in various types of cancer, including prostate cancer. However, to manage these difficulties, prostate cancer cells overexpress GRP78, an endoplasmic reticulum chaperone that enables cancer cells to survive and grow. $^{25,26}$

In this study, the cancer patient cohort contained 443 patients. Of these, 399 patients had Gleason scores ranging from 2 to 6 , and 44 had Gleason scores ranging from 7 to 10. The 44 patients with higher Gleason scores had the highest Shh expression levels (mean histoscore, 172.8), 

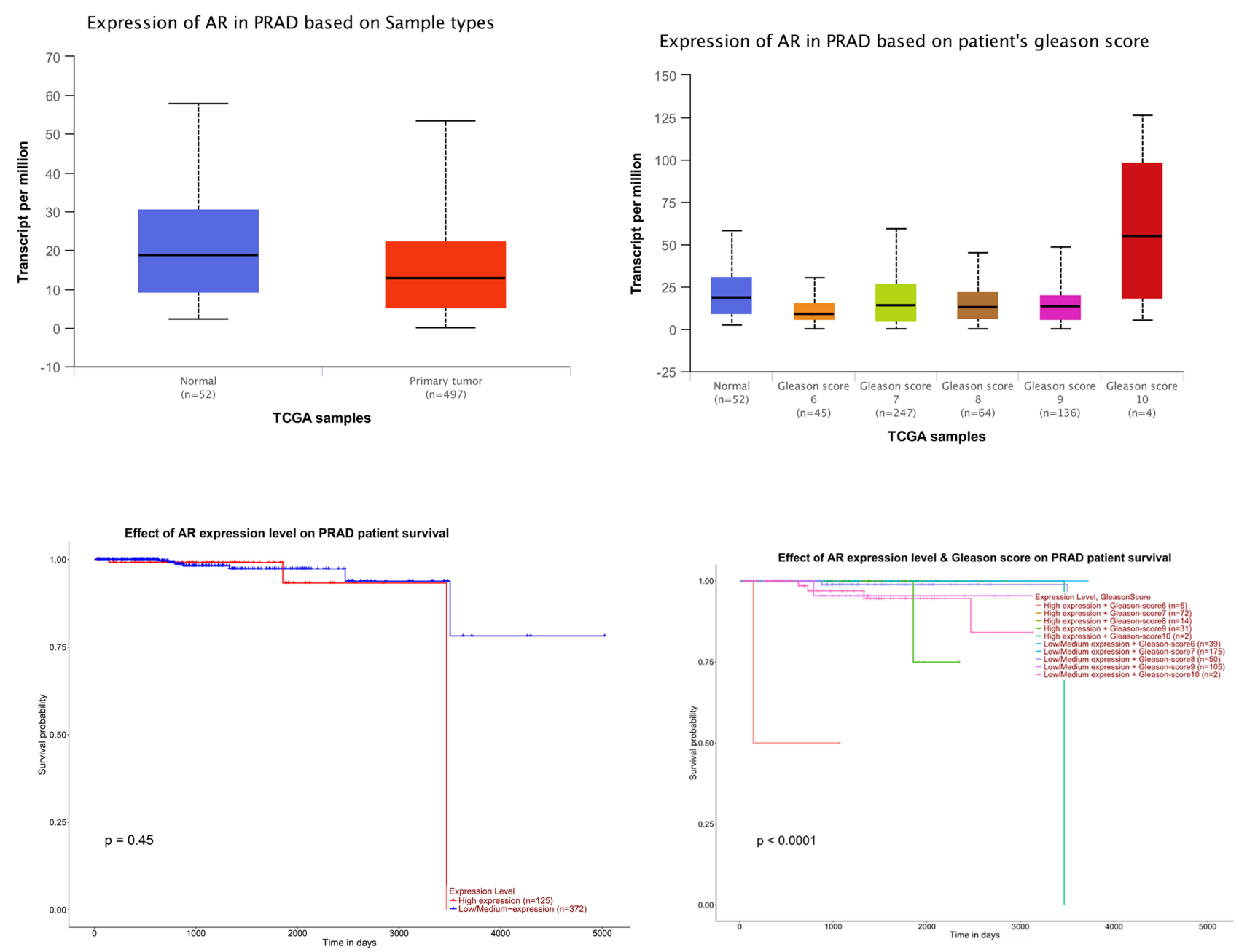

Figure 4 TCGA data comparing the relative expression of AR in normal tissues versus tumor tissues, and survival analysis showed the association of gene expression levels with patients' overall survival.

while those with scores of $2-6$ had a mean histoscore of 147.5, and the control group with benign prostate hyperplasia had a mean histoscore of 58. It seems that Shh expression was positively correlated with the Gleason scores of prostate cancer patients. High Shh expression may be an indicator of poor prognosis, as the survival analysis indicated that $\mathrm{PCa}$ patients had shorter survival than patients with lower Shh expression $(\mathrm{P}<0.05)$. We also noted that Shh was mainly located in the cell membrane and cytoplasm, maybe because that Shh could be secreted out of cancer cells. This result was consistent with a previous study, which reported that Shh was detected in cultured cancer cell media. ${ }^{33}$

We also studied the relationship between the expression of Shh and AR. First, we divided cancer patients into the $\mathrm{AR}$-positive $\left(\mathrm{AR}^{+}\right)$and $\mathrm{AR}$-negative $\left(\mathrm{AR}^{-}\right)$groups. The mean histoscores of Shh in the AR- negative and AR-positive groups were 88 and 154.8, respectively. The correlation coefficient between Shh and AR expression was 0.334, and the difference between the AR-negative and AR-positive groups was significant $(P<0.05)$. This indicated that Shh expression may be influenced by AR directly or indirectly. Prior work has suggested that AR was negatively regulated by SHH signaling. AR has been reported to activate the Gli transcriptional factor and enhance the expression of the Shh signaling pathway. Further, it has been shown that interaction between AR and Gli promotes prostate cancer progression to the CRPC stage, ${ }^{34}$ but the exact mechanism of this interaction requires further study.

GRP78 was usually considered as a poor prognostic indicator for prostate cancer. Moreover, we studied the clinical correlation between GRP78 and Shh. When 

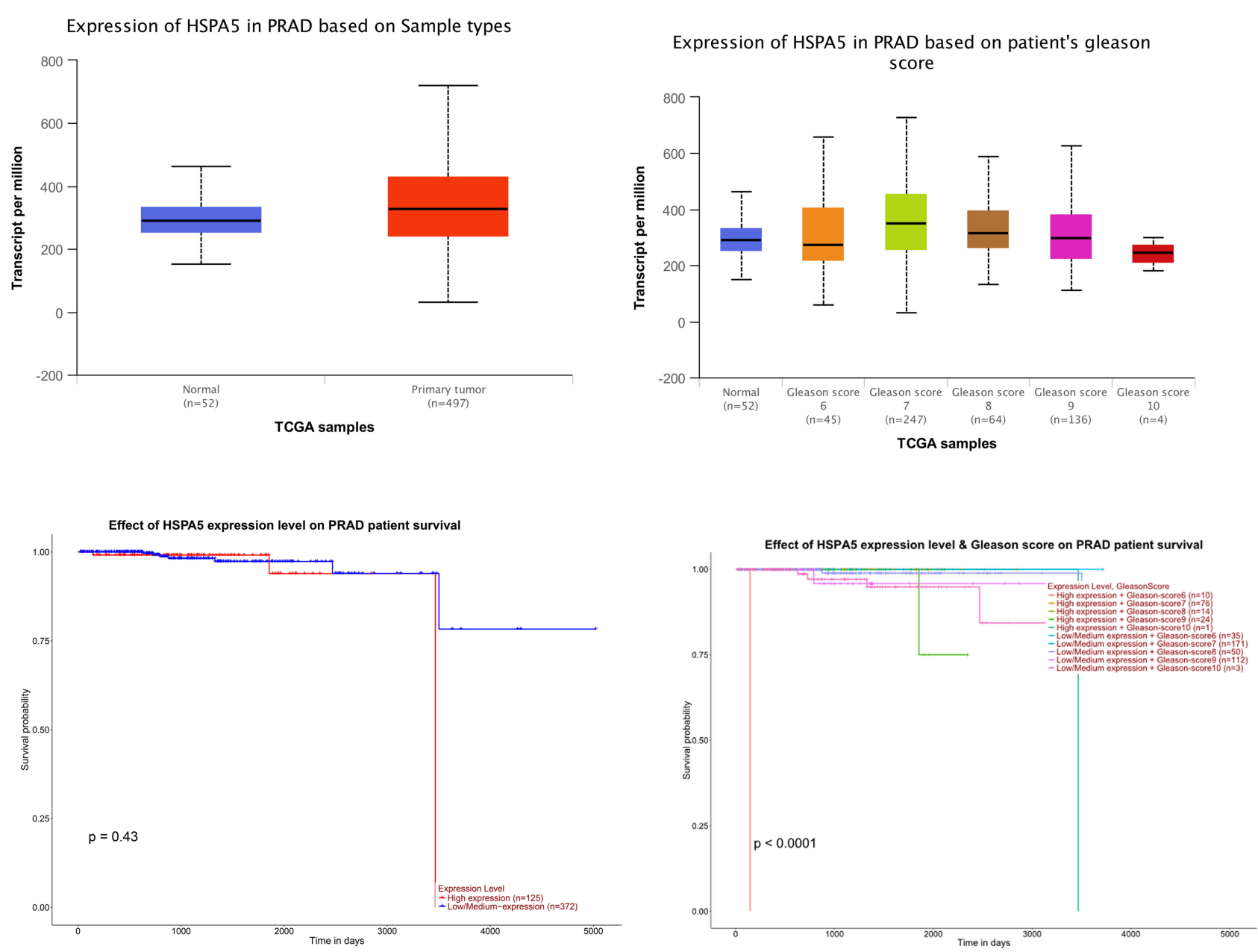

Figure 5 TCGA data comparing the relative expression of GRP78 in normal tissues versus tumor tissues, and survival analysis showed the association of gene expression levels with patients' overall survival.

prostate cancer cells express high levels of GRP78 after chemotherapy, it indicates that chemotherapeutic treatment may be ineffective. ${ }^{35}$ We also observed that GRP78 was expressed in both the cytoplasm and the cancer cell membrane, which is consistent with previous studies. ${ }^{26}$ The mean histoscores of the Shh protein in the GRP78-negative and GRP78-positive groups were 102.7 and 168.5, respectively. The correlation coefficient between Shh and AR expression was 0.227, and the ARnegative and AR-positive groups differed significantly in Shh expression $(\mathrm{P}<0.05)$. The relationship between the expression of GRP78 and AR in prostate cancer is an interesting problem, and we plan to study the possible regulatory mechanisms in our future research. This may be related to $A R$, as one report has suggested that $A R$ can regulate the expression of GRP78. This study focused on the three proteins Shh, AR, and GRP78 in prostate cancer patients using IHC methods, and the concise mechanism of their interactions was not clear. Future work to further elucidate their interactions could provide valuable new information.

As Gleason score is an important indicator in prostate cancer treatment, it can determine which treatment strategies are suitable for a patient. By using the TCGA database, we analyzed the expression of AR, GRP78, Shh in different Gleason score groups, and analyzed the patient survival of different Gleason score groups. The protein expression level was different in the different Gleason score groups, and the patient's survival was also different.

In conclusion, Shh plays multiple important roles in prostate cancer progression and may be regulated by AR. Shh is also closely related to poor survival rates among prostate cancer patients. However, the mechanism underlying its correlation with AR and GRP78 requires further study. This work provides support for future investigation 

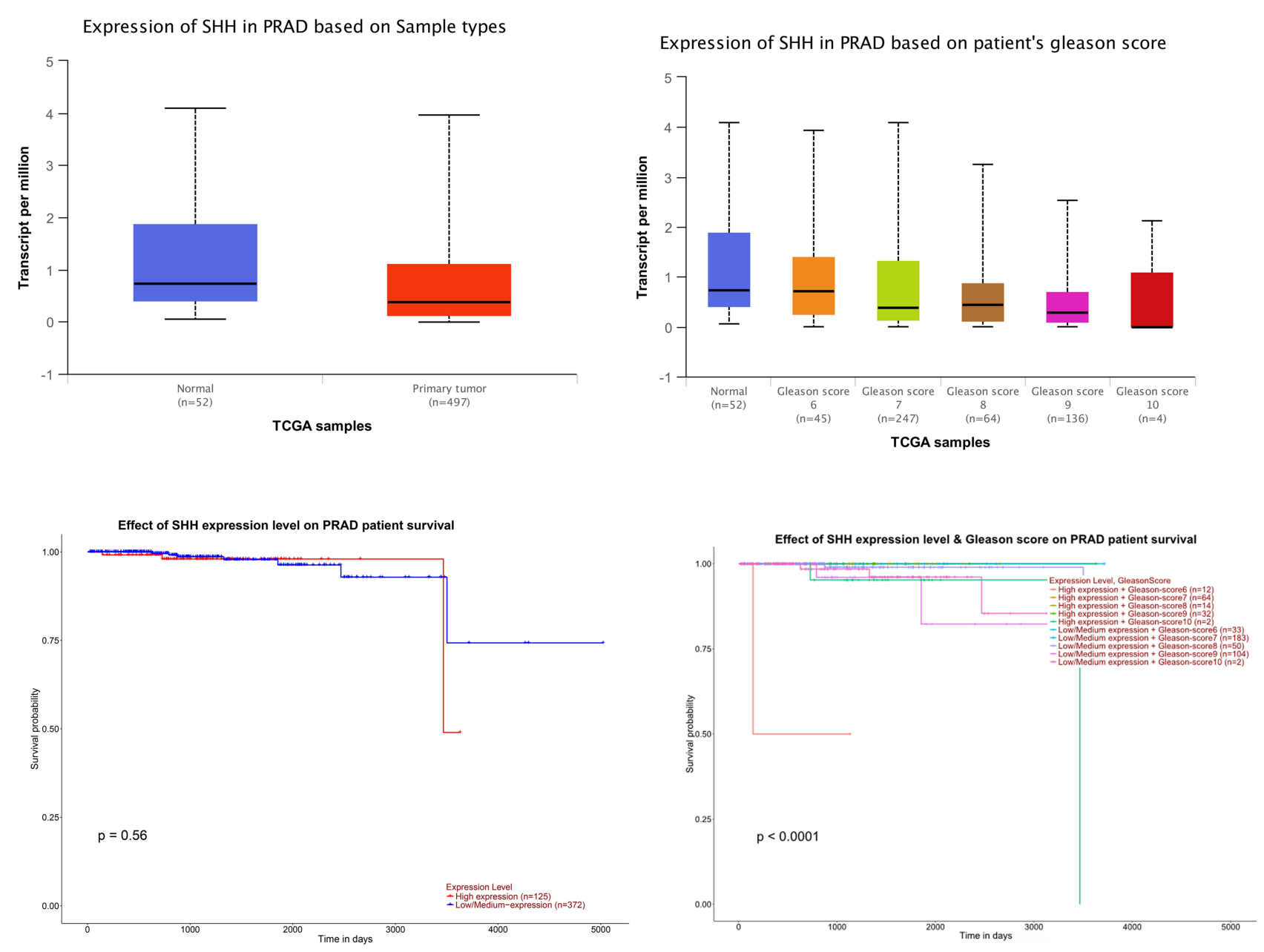

Figure 6 TCGA data comparing the relative expression of Shh in normal tissues versus tumor tissues, and survival analysis showed the association of gene expression levels with patients' overall survival.

of Shh as a potential prognostic marker and therapeutic target.

\section{Abbreviations}

Shh, sonic hedgehog; GRP78, 78 kDa glucose-regulated protein; AR, androgen receptor; ER, endoplasmic reticulum; IHC, immunohistochemistry; PRAD, prostate adenocarcinoma.

\section{Acknowledgment}

This research was supported through grants from the Natural Science Foundation of Shandong Province (No. ZR2017QH005) and the National Natural Science Foundation of China (Nos. 81803097 and 81602727).

\section{Disclosure}

The authors report no conflicts of interest in this work.

\section{References}

1. Bray F, Ferlay J, Soerjomataram I, Siegel RL, Torre LA, Jemal A. Global cancer statistics 2018: GLOBOCAN estimates of incidence and mortality worldwide for 36 cancers in 185 countries. CA Cancer J Clin. 2018;68(6):394-424. doi:10.3322/caac.v68.6

2. Monn MF, Tatem AJ, Cheng L. Prevalence and management of prostate cancer among East Asian men: current trends and future perspectives. Urol Oncol. 2016;34(2):58.e1-9. doi:10.1016/j. urolonc.2015.09.003

3. Vale CL, Burdett S, Rydzewska LHM, et al.; STOpCaP Steering Group. Addition of docetaxel or bisphosphonates to standard of care in men with localised or metastatic, hormone-sensitive prostate cancer: a systematic review and meta-analyses of aggregate data. Lancet Oncol. 2016;17(2):243-256. doi:10.1016/S14702045(15)00489-1

4. Barry MJ, Simmons LH. Prevention of prostate cancer morbidity and mortality: primary prevention and early detection. Med Clin North Am. 2017;101(4):787-806. doi:10.1016/j.mcna.2017.03.009

5. Pezaro C, Woo HH, Davis ID. Prostate cancer: measuring PSA. Intern Med J. 2014;44(5):433-440. doi:10.1111/imj.12407

6. Shah RB, Zhou M. Recent advances in prostate cancer pathology: Gleason grading and beyond. Pathol Int. 2016;66(5):260-272. doi:10.1111/pin. 12398 
7. Kweldam CF 1, van Leenders GJ 1, van der Kwast T 2. Grading of prostate cancer: a work in progress. Histopathology. 2019;74 (1):146-160. doi:10.1111/his.2019.74.issue-1

8. Clarke MG, Wilson JR, Kennedy KP, MacDonagh RP. Clinical judgment analysis of the parameters used by consultant urologists in the management of prostate cancer. J Urol. 2007;178(1):98-102. doi:10. 1016/j.juro.2007.03.029

9. McKenney JK, Wei W, Hawley S, et al. Histologic grading of prostatic adenocarcinoma can be further optimized: analysis of the relative prognostic strength of individual architectural patterns in 1275 patients from the canary retrospective cohort. Am J Surg Pathol. 2016;40 (11):1439-1456. doi:10.1097/PAS.0000000000000736

10. Mahal BA, Berman RA, Taplin ME, Huang FW. Prostate cancer-specific mortality across Gleason scores in black vs nonblack men. JAMA. 2018;320(23):2479-2481. doi:10.1001/jama.2018.11716

11. Saikia B, Gupta K, Saikia UN. The modern histopathologist: in the changing face of time. Diagn Pathol. 2008;3:25. doi:10.1186/17461596-3-25

12. Vollmer E, Goldmann T. Pathology on the edge of interdisciplinarity. A historical epitome. Rom J Morphol Embryol. 2011;52(1 Suppl):223-230.

13. Cao S, Zhan Y, Dong Y. Emerging data on androgen receptor splice variants in prostate cancer. Endocr Relat Cancer. 2016;23(12):T199T210. doi:10.1530/ERC-16-0298

14. Kohli M, Ho Y, Hillman DW, et al. Androgen receptor variant AR-V9 is coexpressed with AR-V7 in prostate cancer metastases and predicts abiraterone resistance. Clin Cancer Res. 2017;23 (16):4704-4715. doi:10.1158/1078-0432.CCR-17-0017

15. Ammirante M, Shalapour S, Kang Y, Jamieson CA, Karin M. Tissue injury and hypoxia promote malignant progression of prostate cancer by inducing CXCL13 expression in tumor myofibroblasts. Proc Natl Acad Sci U S A. 2014;111(41):14776-14781. doi:10.1073/ pnas. 1416498111

16. Shiao SL, Chu GC, Chung LW. Regulation of prostate cancer progression by the tumor microenvironment. Cancer Lett. 2016;380 (1):340-348. doi:10.1016/j.canlet.2015.12.022

17. Leach DA, Need EF, Toivanen R, et al. Stromal androgen receptor regulates the composition of the microenvironment to influence prostate cancer outcome. Oncotarget. 2015;6(18):16135-16150. doi:10.18632/ oncotarget.3873

18. Wu JB, Yin L, Shi C, et al. MAOA-dependent activation of Shh-IL6RANKL signaling network promotes prostate cancer metastasis by engaging tumor-stromal cell interactions. Cancer Cell. 2017;31 (3):368-382. doi:10.1016/j.ccell.2017.02.003

19. Gonnissen A, Isebaert S, Perneel C, et al. Tissue microarray analysis indicates hedgehog signaling as a potential prognostic factor in intermediate-risk prostate cancer. BMC Cancer. 2017;17(1):634. doi:10.1186/s12885-017-3619-4

20. Sanchez P, Hernández AM, Stecca B, et al. Inhibition of prostate cancer proliferation by interference with SONIC HEDGEHOG-GLI1 signaling. Proc Natl Acad Sci U S A. 2004;101(34):12561-12566. doi:10.1073/pnas.0404956101
21. Sheng T, Li C, Zhang X, et al. Activation of the hedgehog pathway in advanced prostate cancer. Mol Cancer. 2004;3:29. doi:10.1186/14764598-3-29

22. Datta S, Datta MW. Sonic Hedgehog signaling in advanced prostate cancer. Cell Mol Life Sci. 2006;63(4):435-448. doi:10.1007/s00018005-5389-4

23. Gagné-Sansfaçon J, Allaire JM, Jones C, Boudreau F, Perreault N. Loss of Sonic hedgehog leads to alterations in intestinal secretory cell maturation and autophagy. PLoS One. 2014;9(6):e98751. doi:10.1371/journal. pone. 0098751

24. Stone S, Ho Y, Li X, et al. Dual role of the integrated stress response in medulloblastoma tumorigenesis. Oncotarget. 2016;7(39):64124-64135. doi:10.18632/oncotarget.11873

25. Gifford JB, Hill R. GRP78 influences chemoresistance and prognosis in cancer. Curr Drug Targets. 2018;19(6):701-708. doi:10.2174/ 1389450118666170615100918

26. Tan SS, Ahmad I, Bennett HL, et al. GRP78 up-regulation is associated with androgen receptor status, Hsp70-Hsp90 client proteins and castrate-resistant prostate cancer. J Pathol. 2011;223(1):81-87. doi:10.1002/path.v223:1

27. Kirkegaard T, Edwards J, Tovey S, et al. Observer variation in immunohistochemical analysis of protein expression, time for a change? Histopathology. 2006;48(7):787-794. doi:10.1111/his.2006.48.issue-7

28. Petrova R, Joyner AL. Roles for Hedgehog signaling in adult organ homeostasis and repair. Development. 2014;141(18):3445-3457. doi: $10.1242 / \mathrm{dev} .083691$

29. Lubik AA, Nouri M, Truong S, et al. Paracrine sonic hedgehog signaling contributes significantly to acquired steroidogenesis in the prostate tumor microenvironment. Int $J$ Cancer. 2017;140 (2):358-369. doi:10.1002/ijc.v140.2

30. Rovida E, Stecca B. Mitogen-activated protein kinases and Hedgehog-GLI signaling in cancer: a crosstalk providing therapeutic opportunities? Semin Cancer Biol. 2015;35:154-167. doi:10.1016/j. semcancer.2015.08.003

31. Rimkus TK, Carpenter RL, Qasem S, Chan M, Lo HW. Targeting the Sonic Hedgehog signaling pathway: review of smoothened and GLI inhibitors. Cancers (Basel). 2016;8(2):E22. doi:10.3390/cancers 8020022

32. Mimeault M, Rachagani S, Muniyan S, et al. Inhibition of hedgehog signaling improves the anti-carcinogenic effects of docetaxel in prostate cancer. Oncotarget. 2015;6(6):3887-3903. doi:10.18632/oncotarget.2932

33. Statkiewicz M, Maryan N, Lipiec A, et al. The role of the SHH gene in prostate cancer cell resistance to paclitaxel. Prostate. 2014;74 (11):1142-1152. doi:10.1002/pros.22830

34. Li N, Truong S, Nouri M, et al. Non-canonical activation of hedgehog in prostate cancer cells mediated by the interaction of transcriptionally active androgen receptor proteins with Gli3. Oncogene. 2018;37(17):2313-2325. doi:10.1038/s41388-017-0098-7

35. Daneshmand S, Quek ML, Lin E, et al. Glucose-regulated protein GRP78 is up-regulated in prostate cancer and correlates with recurrence and survival. Hum Pathol. 2007;38(10):1547-1552.

\section{Publish your work in this journal}

Cancer Management and Research is an international, peer-reviewed open access journal focusing on cancer research and the optimal use of preventative and integrated treatment interventions to achieve improved outcomes, enhanced survival and quality of life for the cancer patient.
The manuscript management system is completely online and includes a very quick and fair peer-review system, which is all easy to use. Visit http://www.dovepress.com/testimonials.php to read real quotes from published authors. 